\title{
Nutritional impact of partial or complete replacement of clover hay by untreated or biologically treated rice straw and corn stalks on: 1. growth performance and economic evaluation of growing New Zealand (NZW) White rabbits
}

Hamed A. A. Omer ${ }^{1}$, Sawsan M. Ahmed ${ }^{1}$, Roshdy I. El-Kady ${ }^{1}$, Aly A. El-Shahat', Mahmoud Y. El-Ayek², Walid S. El-Nattat ${ }^{3 *}$ (I) and Ashraf A. A. Morad ${ }^{1}$

\begin{abstract}
Background: Agriculture by-products are considered a great potential value for utilization by ruminants as well as rabbits. They usually can be the maintenance and part of the production requirements. However, in developing countries, as well as in Egypt, animals suffer from shortage of feeds that are continuously increasing in costs. In general, biological treatments were shown to be the most effective and improved chemical composition of rice straw or corn stalks.

Method: This work aimed to investigate the possible ways of utilizing rice straws or corn stalks in rabbit feeding. The field work is designed to study the effect of biological treatment of Pleurotus ostreatus cultivated on rice straws and Trichoderma reesei cultivated on corn stalks and replacing clover hay by rice straws and corn stalks at levels of $0,33,66$, and $100 \%$ either without or with microbes adding. Seventy-eight New Zealand White (NZW) rabbits aged 4-5 weeks $(565 \pm 13.57 \mathrm{~g})$ were randomly divided into thirteen equal experimental groups.
\end{abstract}

Results: Untreated rice straws or biologically treated with Pleurotus ostreatus increased their contents of crude protein (CP) by 178.75 and $224.5 \%$ and nitrogen-free extract (NFE) by 6.30 and 24.53 , respectively. Meanwhile, crude fiber (CF) content was reduced by 31.32 and 56.75\%, and organic matter content was decreased by 2.81 and 5.51\%, respectively, in comparison with the raw rice straws. Also, biological treatment of rice straws caused a decrease in values of neutral detergent fiber (NDF), acid detergent fiber (ADF), acid detergent lignin (ADL), and hemicellulose contents in comparison with either raw or treated rice straws. Furthermore, biological treatment with Trichoderma reesei realized a decrease in organic matter $(\mathrm{OM}), \mathrm{CF}, \mathrm{NDF}$, and $\mathrm{ADF}$ and increased $\mathrm{CP}$ and ash contents in corn stalks. NFE content of corn stalks was decreased as a result of treatment without or with Trichoderma reesei experimental rations by $11.95 \%$ and $3.82 \%$ compared to raw corn stalks (CS). Biological treatments with fungi significantly $(P<0.05)$ improved average daily gain (ADG) and feed conversion. ADG and feed conversion were significantly $(P<0.05)$ improved when rabbits were fed diets containing rice straw (RS) compared to that fed CS.

(Continued on next page)

\footnotetext{
* Correspondence: elnattat@gmail.com

${ }^{3}$ Animal Reproduction and Artificial Insemination Department, National

Research Centre, 33 El-Bohouth Street, P.O: 12622, Dokki, Giza, Egypt

Full list of author information is available at the end of the article
} 


\begin{abstract}
(Continued from previous page)
Rabbits fed diets replaced clover hay $(\mathrm{CH})$ with $33 \%$ or $66 \%$ of RS or CS significantly increased ADG compared to control and that replaced $100 \%$ of both RS and CS containing rations. Levels of replacing had no significant effect on their dry matter intake (DMI) values. The highest improvement in feed conversion was recorded with rabbits that received diets replaced $33 \%$ of berseem hay $(\mathrm{BH})$ by $\mathrm{RS}$ or $\mathrm{CS}$, followed by that replaced $66 \%$ of $\mathrm{BH}$ by $\mathrm{RS}$ or CS. There were significantly interactions between biological treatments $(T)$, roughage source $(S)$, and replacement levels $(L)(T \times S \times L)$ only on ADG. The best fed conversion was realized by rabbits fed diet replaced $B H$ with $33 \%$ of RS that are treated by Pleurotus ostreatus (4.05 g DMI/g gain). Rabbits fed 33\% biologically treated rice straw with Pleurotus ostreatus showed the highest economic efficiency (179\%) followed by rabbits that received 33\% of both rice straws treated without Pleurotus ostreatus and rabbits that received corn stalks biologically treated with Trichoderma reesei (161\%).
\end{abstract}

Conclusion: Biological treatments of rice straws by Pleurotus ostreatus or corn stalks by Trichoderma reesei were safe, and it improves their chemical analysis and improved both daily gain and feed conversion, decreasing the costing of diet formulation which consequently decreased the price of 1-kg live body weight.

Keywords: Biological treatments, Pleurotus ostreatus, Trichoderma reesei, Rice straw, Corn stalks, Rabbits, Growth performance, Economics

\section{Background}

Agriculture by-products are considered a great potential value for utilization by ruminants as well as rabbits. They represent the maintenance and part of the production requirements (Cheeke 1987). However, in developing countries, as well as in Egypt, animals suffer from shortage of feeds that are continuously increasing in costs.

There is a considerable variation in quantities and qualities of by-products between crops, influenced by species, varieties, climate, season, region, and stage at harvest. The most important parts of roughage are the aerial parts (stems and leaves). These can be utilized as fresh or dry and cut or grazed in the field or in the stable/barn (Chahal 1985).

In Egypt, there are about 25 million tons of plant by-products produced annually. However, wheat straw, rice straw and corn stalks are already being fully utilized by many farmers, either for bedding poultry farms or feeding livestock (Ministry of Agriculture and Land Reclamation 2008). Moreover, Shoukry (2013) recorded that annually, the amount of agricultural residues available in Egypt is about 25.6 million tons, 15.3 million tons of which were crop residues and about 10.3 million tons were agroindustrial by-products. Meanwhile, Sadek (2013) reported that agriculture by-products reached 35 million tons annually, 23 million tons of which were plant wastes ( 7 million tons of them are used as fodders, 4 million tons were used as organic fertilizers, and the rest of wastes (12 million tons) were left without any use). Furthermore, Ali et al. (1987), Abou Akkada (1988), and El-Shinnawy (1990) estimated the quantities of rice straw (2.5 million tons); meanwhile, corn stalks ranged from 3.3 to 3.5 million tons.
Such roughages are usually high in lignocelluloses and low in available energy; also they are generally low in readily available carbohydrates as well as nitrogen and certain minerals. Their utilization is limited by the low voluntary intake of the animals and by their high transport cost, being bulky (Rissanen et al. 1981 and Abedo 2011).

Low-quality roughage includes enormous amount of cereal crop residues such as rice straw, wheat straw, bean straw, maize stover, corn cobs, and rice hulls. These by-products are all characterized by their high fiber, lignin, cellulose, and hemicellulose contents (Goering and Van Soest 1970).

Also, most cereal wastes are characterized by low crude protein, low available energy, and deficiency in certain minerals. These low-quality roughages are inefficiently utilized by ruminants. This is due to low digestibility and poor nutritive value associated particularly with cereal straw. Their utilization is also limited because of low voluntary intake of the animals and their huge bulk, which makes transportation more expensive. These materials supply no more energy than poor quality hay; their total digestible nutrients (TDN) are less than 50\%, and starch value is less than $29 \%$ (Balch 1976).

Chahal (1985) noted that the chemical composition of field crop residues contains about $30-40 \%$ cellulose, 16$27 \%$ hemicelluloses, and 3-13\% lignin.

Regarding the world feed shortage, a great deal of research works was directed not only to make full use of the roughages but also to increase their feeding value. The intake and utilization of low-quality roughages could be increased by proper supplementation or by various pretreatments. Such methods may involve physical, chemical, and microbial treatments and improve digestibility and nutritive values of field crop residues and wastes (Rangnekar et al. 1982 and Cheeke 1987). 
In general, biological treatments were shown to be the most effective way to improve the chemical composition of rice straw or corn stalks (Kiran Singh 1991; Krishna Moorthy et al. 1996; Rangaswami and Bagyaraj 1996; Mahrous and Abou Ammou 2005; Abd-Allah 2007; Abo-Eid et al. 2007; Abd El-Galil 2008).

So, this work was carried out to investigate the impact of feeding the biologically treated or untreated rice straw and corn stalks using Pleurotus ostreatus or Trichoderma reesei at different levels $(0,33,66$, and $100 \%)$ on growth performance and economic efficiency of growing NZW rabbits.

\section{Methods}

The present study was carried out at El-Nubaria Experimental and Production Station, Rabbit Research Unit, El-Imam Malik Village, National Research Centre.

This work aimed to investigate the possible ways of utilizing rice straw or corn stalks in rabbit feeding. The field work is designed to study the effect of biological treatment by Pleurotus ostreatus cultivated on rice straw and Trichoderma reesei cultivated on corn stalks and replacing clover hay by rice straw and corn stalks at levels $(0,33,66$, and $100 \%$ ) either with or without microbes adding.

Seventy-eight immature apparently healthy NZW rabbits aged $4-5$ weeks $(565 \pm 13.57 \mathrm{~g})$ were randomly divided into 13 equal experimental groups (6 rabbit in each treatment).

Weaned rabbits were housed in galvanized wire cages $(50 \times 50 \times 45 \mathrm{~cm})$ and provided with stainless steel nipples for drinking, and the feeders allow the recording of feed intake during the feeding trial that continue 13 weeks (91 days).

All experimental groups were kept under the same managerial conditions, and rations were offered pelleted with diameter $4 \mathrm{~mm}$.

Live weight of rabbits was individually recorded weekly, and average daily gain was calculated. Also, feed intake was also recorded weekly throughout the 13 weeks of feeding trial. Feed conversion expressed as $g$ dry matter intake/g gain was calculated.

\section{Biological treatments Microorganisms}

Pleurotus ostreatus and Trichoderma reesei were obtained from the Agriculture Microbiology Department, National Research Centre, Egypt.

\section{Preparation of fungal inoculums}

Three-day-old slant cultures of Pleurotus ostreatus or Trichoderma reesei were crushed into flasks containing $20 \mathrm{ml}$ of sterilized water. The fungi spore suspensions were used as inoculums at $10 \% \mathrm{v} / \mathrm{w}$ to inoculate $500 \mathrm{ml}$ capacity flasks containing $25 \mathrm{~g}$ of ground waste moistened at solid:liquid ratio of 1:2 with basal medium composed by g/L, 40 sugar cane molasses, 2.0 urea, 0.2 potassium dihydrogen phosphate, and 0.3 magnesium sulfate; the inoculated flasks were incubated at $30{ }^{\circ} \mathrm{C}$ for $72 \mathrm{hrs}$ under static solid-state fermentation system.

\section{Preparation of fungal biological treatments}

The above prepared inoculate was employed to inoculate $500 \mathrm{~g}$ of crop residues under study moistened by the above basal medium at solid:liquid (1:2) at $10 \% \mathrm{v} / \mathrm{w}$ and packed in polyethylene bags $(50 \times 100 \mathrm{~cm})$. The inoculated bags were incubated under static conditions for 10 days at $30 \pm 2{ }^{\circ} \mathrm{C}$; at the end of incubation period, the bags were opened and oven dried at $70{ }^{\circ} \mathrm{C}$ till constant weight and the residues are milled for chemical analysis purpose.

\section{Preparation of substrate}

A total of $25.00 \mathrm{~g}$ of the dried milled substrate were weighed and transferred into a dry clean sterile bottle (120 ml capacity); then $70 \mathrm{ml}$ distilled water were added. Each bottle was immediately coerced with aluminum foil and sterilized in the autoclave at $121{ }^{\circ} \mathrm{C}$ for $15 \mathrm{~min}$. The treatments were run in triplicates.

\section{Inoculation}

Each bottle was inoculated at the center of the substrate with 2 mycelia disc (10 mm diameter) and covered immediately. They were kept in the dark cupboard in the laboratory at $30{ }^{\circ} \mathrm{C}$ and $100 \%$ relative humidity (RH). After 21 days of inoculation, the experimental bottles were harvested by autoclaving again to terminate the mycelia growth. Samples of the biodegraded samples were oven dried to constant weight for chemical analysis.

\section{Biological treatments of crop residues under study in large scale}

A heap of $160 \mathrm{~kg}$ of each tested chopped and crushed rice straw or corn stalks were moistened with medium containing $2.5 \%$ molasses, $2.5 \%$ urea, $1.5 \%$ ammonium sulfate, $1.00 \%$ supper phosphate, and $0.5 \%$ magnesium sulfate at solid:liquid (1:2). About $80 \mathrm{~kg}$ of each crop residues were used with the mixture of fermented fungal biomass and mixed well in mineral medium container and spread and mixed well on a plastic sheet which contained the crop residues. The treated crop residues were shuffled up and downside daily for the prop inoculation period (14 days). At the end of fermentation period, the treated crop residues were collected and exposed to sundry until the moisture content reached $>10 \%$ and then packed and stored until used in manufacturing the pelleted feed (Fadel et al., 1992). 


\section{Manufacturing the pelleted feed}

The air-dried compost have been transported to the forage manufacture for making the experimental pelleted feed by substituting clover hay with biologically treated and untreated rice straw or corn stalks at the levels of 33,66 , and $100 \%$.

\section{Animals and feeds}

A total number of 78 weaned NZW rabbits were randomly divided into 13 experimental groups of 6 rabbits each. Each group is divided into three replicates of two rabbits each.

All the experimental diets were formulated to be approximately iso-nitrogenous and isocaloric that meet requirements of growing rabbits according to NRC (1977) recommendation.

The ingredients used in diet formulation were shown in Table 1. Meanwhile, the composition and chemical analysis of the experimental diets were presented in Tables 2 and 3.

The first rations contained 33\% clover hay $(\mathrm{CH})$ from the total diet formulation, and it is considered as the control. Rations from 2 to 7 had replaced $\mathrm{CH}$ by rice straw without and with Pleurotus ostreatus, and rations from 8 to 13 had replaced $\mathrm{CH}$ by corn stalks without and with Trichoderma reesei.

All animals were kept under the same managerial and hygienic conditions. Each replicate which involved two rabbits was housed separately in metal cages and provided with feed and water ad libitum. Ambient temperature at $27-28{ }^{\circ} \mathrm{C}$ was achieved with natural light and good ventilation.
Chemical analysis of $\mathrm{CH}$; rice straw (raw, untreated, or treated with Pleurotus ostreatus), corn stalks (raw, untreated or treated with Trichoderma reesei), and the experimental diets was determined according to AOAC (2005) methods.

\section{Economic efficiency}

Economic efficiency of the experimental rations for body weight gain and the cost of feed required for producing $1 \mathrm{~kg}$ of body weight gain were calculated. The costs of experimental diets were calculated according to the price prevailing at local market as well as the price of tested materials (rice straw and corn stalks) at the time of experiment.

Total feed cost $=$ average feed consumption $\mathrm{kg} \times$ price per $1-\mathrm{kg}$ feed

Cost feed per kg gain = total feed cost $/$ weight gain

Total revenue $=$ weight gain $\times$ price per $\mathrm{kg}$ live body weight

Net revenue $=$ price of weight gain - total feed cost

Economic efficiency $=$ net revenue $/$ total feed cost

\section{Analytical procedures}

Chemical analysis of ingredients and the experimental rations were analyzed according to AOAC (2005). NDF, $\mathrm{ADF}$, and ADL were determined according to Goering and Van Soest (1970) and Van Soest et al. (1991). Hemicellulose and cellulose contents were calculated by difference using the following equations:

Hemicellulose $=$ NDF-ADF

Cellulose $=\mathrm{ADF}-\mathrm{ADL}$

Table 1 Chemical analysis of different roughages used in ration formulations

\begin{tabular}{|c|c|c|c|c|c|c|c|}
\hline \multirow[t]{2}{*}{ Item } & \multirow{2}{*}{$\begin{array}{l}\text { Clover } \\
\text { hay } \\
(\mathrm{CH})\end{array}$} & \multicolumn{3}{|c|}{ Rice straw (RS) } & \multicolumn{3}{|c|}{ Corn stalks (CS) } \\
\hline & & Raw & Without Pleurotus ostreatus & With Pleurotus ostreatus & Raw & Without Trichoderma reesei & With Trichoderma reesei \\
\hline Moisture \% & 13.88 & 6.70 & 7.50 & 8.00 & 7.00 & 8.50 & 8.20 \\
\hline \multicolumn{8}{|c|}{ Chemical analysis on DM basis\% } \\
\hline OM & 89.10 & 89.00 & 86.50 & 84.10 & 88.50 & 87.01 & 84.69 \\
\hline $\mathrm{CP}$ & 17.03 & 5.27 & 14.69 & 17.10 & 5.61 & 13.99 & 16.68 \\
\hline CF & 14.07 & 45.66 & 31.36 & 19.75 & 37.78 & 32.96 & 24.13 \\
\hline $\mathrm{EE}$ & 2.50 & 1.10 & 1.15 & 1.21 & 1.11 & 1.32 & 1.56 \\
\hline NFE & 55.50 & 36.97 & 39.30 & 46.04 & 44.00 & 38.74 & 42.32 \\
\hline Ash & 10.90 & 11.00 & 13.50 & 15.90 & 11.50 & 12.99 & 15.31 \\
\hline \multicolumn{8}{|c|}{ Cell wall constituents \% } \\
\hline NDF & 33.84 & 68.75 & 65.75 & 61.99 & 72.50 & 65.85 & 60.00 \\
\hline ADF & 18.17 & 47.50 & 45.15 & 43.75 & 56.00 & 47.75 & 45.25 \\
\hline $\mathrm{ADL}$ & 12.89 & 14.85 & 13.56 & 10.99 & 15.56 & 16.95 & 18.75 \\
\hline Hemicellulose & 15.67 & 21.25 & 20.60 & 18.24 & 16.50 & 18.10 & 14.75 \\
\hline Cellulose & 5.28 & 32.65 & 31.59 & 32.76 & 40.44 & 30.80 & 26.50 \\
\hline
\end{tabular}

Hemicellulose $=N D F-A D F$. Cellulose $=A D F-A D L$ 
Table 2 Composition of the experimental rations

\begin{tabular}{|c|c|c|c|c|c|c|c|c|c|c|c|c|c|}
\hline \multirow[t]{4}{*}{ Ingredients } & \multirow{4}{*}{$\begin{array}{l}\text { Clover } \\
\text { hay } \\
\text { (CH) } \\
\text { control } \\
0 \% \\
\mathrm{R}_{1} \\
\end{array}$} & \multicolumn{6}{|c|}{ Rice straw (RS) } & \multicolumn{6}{|c|}{ Corn stalks (CS) } \\
\hline & & \multicolumn{3}{|c|}{ Without Pleurotus ostreatus } & \multicolumn{3}{|c|}{ With Pleurotus ostreatus } & \multicolumn{3}{|c|}{ Without Trichoderma reesei } & \multicolumn{3}{|c|}{ With Trichoderma reesei } \\
\hline & & $33 \%$ & $66 \%$ & $100 \%$ & $33 \%$ & $66 \%$ & $100 \%$ & $33 \%$ & $66 \%$ & $100 \%$ & $33 \%$ & $66 \%$ & $100 \%$ \\
\hline & & $\mathrm{R}_{2}$ & $R_{3}$ & $\mathrm{R}_{4}$ & $\mathrm{R}_{5}$ & $R_{6}$ & $\mathrm{R}_{7}$ & $\mathrm{R}_{8}$ & $\mathrm{R}_{9}$ & $\mathrm{R}_{10}$ & $\mathrm{R}_{11}$ & $\mathrm{R}_{12}$ & $\mathrm{R}_{13}$ \\
\hline Clover hay & 33.00 & 22.00 & 11.00 & - & 22.00 & 11.00 & - & 22.00 & 11.00 & - & 22.00 & 11.00 & - \\
\hline Yellow corn & 15.75 & 21.25 & 28.75 & 38.75 & 21.25 & 28.75 & 38.75 & 31.00 & 39.35 & 38.75 & 31.00 & 39.35 & 38.75 \\
\hline Barley & 14.50 & 14.50 & 13.50 & 2.50 & 14.50 & 13.50 & 2.50 & 2.00 & 1.50 & 1.50 & 2.00 & 1.50 & 1.50 \\
\hline Soybean meal & 17.40 & 18.40 & 19.65 & 20.65 & 18.00 & 19.65 & 20.65 & 19.65 & 21.65 & 21.65 & 19.0 .65 & 18.00 & 17.00 \\
\hline Wheat bran & 16.50 & 9.50 & 1.25 & 0.60 & 10.00 & 1.25 & 0.60 & 11.00 & 0.60 & 0.60 & 11.00 & 4.25 & 5.25 \\
\hline Rice straw & - & 11.00 & 22.00 & 33.00 & 11.00 & 22.00 & 33.00 & - & - & - & - & - & - \\
\hline Corn stalks & - & - & - & - & - & - & - & 11.00 & 22.00 & 33.00 & 11.00 & 22.00 & 33.00 \\
\hline Calcium di-phosphate & 2.05 & 2.50 & 2.85 & 3.05 & 2.50 & 2.85 & 3.05 & 2.40 & 2.85 & 3.05 & 2.40 & 2.85 & 3.05 \\
\hline Limestone & 0.10 & 0.10 & 0.20 & 0.60 & 0.10 & 0.20 & 0.60 & 0.20 & 0.25 & 0.60 & 0.20 & 0.25 & 0.60 \\
\hline Sodium chloride & 0.40 & 0.40 & 0.40 & 0.40 & 0.40 & 0.40 & 0.40 & 0.40 & 0.40 & 0.40 & 0.40 & 0.40 & 0.40 \\
\hline Premix* & 0.30 & 0.30 & 0.30 & 0.30 & 0.30 & 0.30 & 0.30 & 0.30 & 0.30 & 0.30 & 0.30 & 0.30 & 0.30 \\
\hline D.L methionine & - & 0.05 & 0.10 & 0.15 & 0.04 & 0.10 & 0.15 & 0.05 & 0.10 & 0.15 & 0.05 & 0.10 & 0.15 \\
\hline Price, LE/ton & 2360 & 2325 & 2302 & 2216 & 2326 & 2322 & 2246 & 2329 & 2323 & 2195 & 2339 & 2284 & 2136 \\
\hline
\end{tabular}

${ }^{*}$ Each $\mathrm{kg}$ of vitamin and mineral mixture contains Vit. A $2.000-000 \mathrm{lu}$, Vit. B $0.33 \mathrm{~g}$, Vit. B 1.09, Vit. B 150.00 lu, Vit. E $8.33 \mathrm{~g}$, Vit K $0.33 \mathrm{~g}$, pantothemic acid $3.33 \mathrm{~g}$, nicatonic acid $30.00 \mathrm{~g}$, Vit. $B_{6} 2.00 \mathrm{~g}$, Vit. $B_{12} 1.7 \mathrm{mg}$, folic acid 0.039 , biotin 33 mg., Cu $0.50 \mathrm{~g}$, choline chloride $200 \mathrm{mg}$, Mn $5.0 \mathrm{~g}$; Fe $12.5 \mathrm{~g}$, Mg 66.7 mg, Co 1.33 $\mathrm{mg}$, Se $16.6 \mathrm{mg}, \mathrm{Zn} 11.9$, iodine $16.6 \mathrm{mg}$, and antioxidant $10.0 \mathrm{~g}$

\section{Statistical analysis}

Data collected were statistically analyzed as three factors factorial analysis of variance using the general linear model procedure of SPSS (2008).

Meanwhile, Duncan's Multiple Range Test was used to examine the significance between means (Duncan (1955)).

The following model was used as the following:

$$
\begin{aligned}
\mathbf{Y}_{\mathbf{i j k} \mathbf{l}=} & \boldsymbol{\mu}+\mathbf{T}_{\mathbf{i}}+\mathbf{S}_{\mathbf{j}}+\mathbf{L}_{\mathbf{k}}+(\mathbf{T S})_{\mathbf{i j}}+(\mathbf{T L})_{\mathbf{i k}}+(\mathbf{S L})_{\mathbf{j k}} \\
& +(\mathbf{T S L})_{\mathbf{i j k}}+\mathbf{e}_{\mathbf{i j k} \mathbf{l}}
\end{aligned}
$$

where $\mathrm{Y}_{\mathrm{ijkl}}=$ observation

$\mu=$ the overall mean

$\mathrm{T}_{\mathrm{i}}=$ the effect of treatment $(\mathrm{T})$ for $\mathrm{i}=1$ to $2,1=\mathrm{un}$ treated (without Pleurotus ostreatus or Trichoderma reesei and 2 = treated biologically (treated with Pleurotus ostreatus or Trichoderma reesi)

$\mathrm{S}_{\mathrm{j}}=$ the effect of roughage $\operatorname{source}(\mathrm{S})$ for $\mathrm{j}=1$ to 2,1 $=$ rice straw and $2=$ corn stalks

$\mathrm{L}_{\mathrm{k}}=$ the effect of level of supplementation ( $\mathrm{L}$ ) for $\mathrm{k}=$ 1 to $4,1=$ control diet containing $33 \%$ clover hay $(0 \%$ replacement) $2=$ replaced $33 \%$ of clover hay by rice straw or corn stalks, 3 = replaced $66 \%$ of clover hay by rice straw or corn stalks, and $4=$ complete replacement (100\%) of clover hay by rice straw or corn stalks

$(\mathrm{TS})_{\mathrm{ij}}=$ the interaction between treatment $(\mathrm{T})$ and roughage sources $(\mathrm{S})$
$(\mathrm{TL})_{\mathrm{ik}}=$ the interaction between treatment $(\mathrm{T})$ and replacement levels (L)

$(\mathrm{SL})_{\mathrm{jk}}=$ the interaction between roughage sources $(\mathrm{S})$ and replacement levels (L)

$(\mathrm{TSL})_{\mathrm{ijk}}=$ the interaction between treatment $(\mathrm{T})$; roughage source $(\mathrm{S})$ and replacement levels (L)

$\mathrm{e}_{\mathrm{ijkl}}=$ the experimental error

\section{Results}

Chemical analysis of different ingredients

Data presented in Table 1 cleared that untreated rice straw or biologically treated with Pleurotus ostreatus showed an increase in their contents of crude protein (178.75 and 224.5\%), NFE (6.30 and 24.53.5\%), and ash (22.73 and $44.45 \%)$ compared to the raw rice straw. On the other hand, CF content is reduced by 31.32 and $56.75 \%$; meanwhile OM content decreased by 2.81 and $5.51 \%$, respectively.

Biological treatment with Trichoderma reesei realized a decrease in OM, CF, NDF, and ADF and increased CP and ash contents in corn stalks (Table 1).

\section{Composition and chemical analysis of the experimental rations}

Data presented in Tables 2 and 3 mentioned that all tested diets were formulated to cover the rabbit's requirements according to NRC (1977). Different experimental diets were approximately iso-nitrogenous and isocaloric. The corresponding values of crude protein 
Table 3 Chemical analysis of the experimental rations

\begin{tabular}{|c|c|c|c|c|c|c|c|c|c|c|c|c|c|}
\hline \multirow[t]{3}{*}{ Ingredients } & \multirow{3}{*}{$\begin{array}{l}\text { Clover } \\
\text { hay } \\
\text { (CH) } \\
\text { control } \\
0 \%\end{array}$} & \multicolumn{6}{|c|}{ Rice straw (RS) } & \multicolumn{6}{|c|}{ Corn stalks (CS) } \\
\hline & & \multicolumn{3}{|c|}{ Without Pleurotus ostreatus } & \multicolumn{3}{|c|}{ With Pleurotus ostreatus } & \multicolumn{3}{|c|}{ Without Trichoderma reesei } & \multicolumn{3}{|c|}{ With Trichoderma reese } \\
\hline & & $33 \%$ & $66 \%$ & $100 \%$ & $33 \%$ & $66 \%$ & $100 \%$ & $33 \%$ & $66 \%$ & $100 \%$ & $33 \%$ & $66 \%$ & $100 \%$ \\
\hline Moisture & 13.88 & 10.05 & 9.78 & 90.83 & 7.38 & 9.17 & 7.52 & 8.79 & 8.90 & 8.89 & 8.46 & 8.57 & 9.03 \\
\hline \multicolumn{14}{|c|}{ Chemical analysis on dry matter (\%) } \\
\hline Organic matter (OM) & 74.25 & 88.11 & 87.45 & 86.03 & 89.24 & 86.08 & 83.76 & 90.14 & 89.86 & 88.45 & 88.98 & 88.64 & 87.93 \\
\hline Crude protein (CP) & 17.03 & 17.05 & 17.02 & 17.03 & 17.21 & 17.21 & 17.76 & 16.99 & 17.02 & 17.09 & 18.11 & 16.95 & 16.57 \\
\hline Crude fiber (CF) & 14.07 & 13.39 & 12.59 & 12.16 & 12.20 & 10.76 & 8.58 & 13.37 & 12.56 & 12.56 & 11.38 & 10.36 & 10.07 \\
\hline Ether extract (EE) & 2.50 & 2.36 & 2.23 & 2.29 & 2.35 & 2.15 & 2.30 & 2.56 & 2.39 & 2.28 & 2.57 & 2.32 & 2.50 \\
\hline Nitrogen-free extract & 40.65 & 55.31 & 55.61 & 54.55 & 57.48 & 55.96 & 55.12 & 57.22 & 57.89 & 56.52 & 56.92 & 59.01 & 58.79 \\
\hline (NFE) Ash & 25.75 & 11.89 & 12.55 & 13.97 & 10.76 & 13.92 & 16.24 & 9.86 & 10.14 & 11.55 & 11.02 & 11.36 & 12.07 \\
\hline \multicolumn{14}{|l|}{ Calculated* } \\
\hline Digestible energy (DE) & 2513 & 2496 & 2491 & 2453 & 2493 & 2445 & 2444 & 2503 & 2595 & 2412 & 2518 & 2501 & 2425 \\
\hline Calcium (Ca) & 1.08 & 1.03 & 0.98 & 1.02 & 0.90 & 0.92 & 0.97 & 1.04 & 1.01 & 1.03 & 0.97 & 0.92 & 0.96 \\
\hline Tryptophane & 0.80 & 0.82 & 0.79 & 0.80 & 0.85 & 0.80 & 0.82 & 0.80 & 0.79 & 0.80 & 0.82 & 0.79 & 0.83 \\
\hline Lysine & 0.89 & 0.83 & 0.77 & 0.72 & 0.86 & 0.40 & 0.71 & 0.85 & 0.80 & 0.74 & 0.85 & 0.71 & 0.63 \\
\hline Methionine + cestine & 0.55 & 0.55 & 0.56 & 0.57 & 0.55 & 0.53 & 0.57 & 0.57 & 0.57 & 0.58 & 0.56 & 0.46 & 0.54 \\
\hline Sodium & 0.20 & 0.19 & 0.18 & 0.17 & 0.19 & 0.79 & 0.18 & 0.19 & 0.18 & 0.17 & 0.17 & 0.18 & 0.17 \\
\hline \multicolumn{14}{|l|}{ Cell wall constituents \% } \\
\hline Neutral detergent fiber (NDF) & 33.84 & 30.32 & 28.49 & 25.29 & 24.02 & 28.60 & 26.28 & 30.46 & 32.12 & 28.49 & 37.97 & 35.24 & 31.68 \\
\hline Acid detergent fiber (ADF) & 18.17 & 14.42 & 15.83 & 14.18 & 13.95 & 13.32 & 13.49 & 14.74 & 17.72 & 14.73 & 17.54 & 15.38 & 16.90 \\
\hline Acid detergent lignin (ADL) & 12.89 & 10.83 & 5.40 & 3.63 & 11.59 & 6.12 & 2.81 & 3.84 & 7.22 & 2.99 & 6.52 & 8.56 & 5.88 \\
\hline Hemicellulose $e^{* *}$ & 15.67 & 15.90 & 13.11 & 11.11 & 10.07 & 15.28 & 12.79 & 15.72 & 14.40 & 13.76 & 20.43 & 19.86 & 14.78 \\
\hline Cellulose ${ }^{* * *}$ & 5.28 & 3.59 & 10.43 & 10.50 & 2.36 & 7.20 & 10.68 & 10.90 & 10.50 & 11.74 & 11.02 & 6.82 & 11.02 \\
\hline
\end{tabular}

*Calculated according to NRC 1977. ${ }^{* * H e m i c e l l u l o s e ~}=$ NDF - ADF. ${ }^{* * *}$ Cellulose $=$ ADF - ADL

(CP) ranged from 16.57 to $18.11 \%$, crude fiber (CF) 8.58 to $14.07 \%$, ether extract (EE) 2.15 to $2.57 \%$, nitrogen free extract (NFE) 40.65 to $59.01 \%$, digestible energy (DE) 2412 to $2595 \mathrm{kcal} / \mathrm{kg}$ feed, neutral detergent fiber (NDF) 24.02 to $37.97 \%$, acid detergent fiber (ADF) 13.32 to $18.17 \%$, acid detergent lignin (ADL) 2.81 to $12.89 \%$, hemicellulose 10.07 to $20.43 \%$, and cellulose 2.36 to $11.74 \%$, among the different tested diets.

\section{Growth performance}

\section{Effect of biological treatments}

Biological treatments with fungi significantly $(P<0.05)$ increased the final body weight $(\mathrm{FW})$, total body weight gain (TBWG), and ADG. In addition, the feed conversion expressed as g DMI/g gain was also significantly $(P$ $<0.05$ ) improved in comparison to rabbits that received rations without fungi (Table 4). Meanwhile, DMI was not affected.

\section{Effect of roughage sources}

TBWG and ADG significantly $(P<0.05)$ improved rabbits fed diets containing RS comparing to that fed CS; however,
FW was nonsignificantly increased with rabbits fed rations containing RS in comparison with those fed rations containing CS. Also, feed conversion was improved $(P<0.05)$ when rabbits fed diets containing RS compared to that fed CS. On the other hand, rabbits that received diets containing RS had nonsignificantly decreased the DMI compared to that fed diets containing CS (Table 4).

\section{Effect of replacement levels}

Data in Table 4 reported that rabbits fed diets replaced $\mathrm{CH}$ in control diet with 33 or $66 \%$ of RS or CS significantly increased their FW, TBWG, and ADG compared to the the control and that replaced $100 \%$ of both RS and CS containing diets; however rabbits that offered diets replaced $100 \%$ of $\mathrm{CH}$ by RS or CS caused a significant decrease in their TBWG and ADG compared to the control. Levels of replacing had no significant effect on their DMI. The highest improvement in feed conversion was recorded with rabbits that received diets replaced $33 \%$ of $\mathrm{CH}$ by RS or CS, followed by that replaced $66 \%$ of $\mathrm{CH}$ by RS or CS. 
Table 4 Main effect of biological treatments, roughage sources and replacement levels on growth performance of the experimental groups

\begin{tabular}{|c|c|c|c|c|c|c|c|c|c|c|c|}
\hline \multirow[t]{2}{*}{ Item } & \multicolumn{3}{|c|}{ Biological treatments } & \multicolumn{3}{|c|}{ Roughage sources } & \multicolumn{5}{|c|}{ Replacement levels } \\
\hline & $\begin{array}{l}\text { Without } \\
\text { fungi }\end{array}$ & $\begin{array}{l}\text { With } \\
\text { fungi }\end{array}$ & SEM & $\begin{array}{l}\text { Rice straw } \\
\text { (RS) }\end{array}$ & $\begin{array}{l}\text { Corn stalks } \\
\text { (CS) }\end{array}$ & SEM & $0 \%$ & $33 \%$ & $66 \%$ & $100 \%$ & SEM \\
\hline Initial weight (IW), g & 570 & 560 & 13.57 & 568 & 562 & 13.57 & 566 & 565 & 570 & 559 & 13.57 \\
\hline Final weight (FW), g & $2089^{b}$ & $2246^{a}$ & 21.82 & 2179 & 2157 & 21.82 & $2082^{c}$ & $2364^{a}$ & $2221^{b}$ & $2004^{c}$ & 21.82 \\
\hline Total body weight gain (TBWG), g & $1519^{b}$ & $1686^{a}$ & 17.55 & $611^{a}$ & $1595^{b}$ & 17.55 & $1516^{c}$ & $1799^{\mathrm{a}}$ & $1651^{b}$ & $1445^{\mathrm{d}}$ & 17.55 \\
\hline Duration period & 91 days & & & & & & & & & & \\
\hline Average daily gain (ADG), g & $16.69^{b}$ & $18.53^{\mathrm{a}}$ & 0.19 & $17.70^{\mathrm{a}}$ & $17.53^{b}$ & 0.19 & $16.66^{\mathrm{c}}$ & $19.77^{\mathrm{a}}$ & $18.14^{b}$ & $15.88^{\mathrm{d}}$ & 0.19 \\
\hline Dry matter intake (DMI), g & 89.62 & 89.26 & 1.38 & 87.20 & 91.68 & 1.38 & 88.69 & 89.17 & 90.02 & 89.90 & 1.38 \\
\hline Feed conversion expressed as (g DM intake) & $5.40^{\mathrm{a}}$ & $4.89^{b}$ & 0.09 & $4.98^{b}$ & $5.30^{\mathrm{a}}$ & 0.09 & $5.23^{b}$ & $4.53^{c}$ & $5.04^{b}$ & $5.71^{\mathrm{a}}$ & 0.09 \\
\hline
\end{tabular}
g gain)

$\mathrm{a}, \mathrm{b}, \mathrm{c}$, and $\mathrm{d}$ : means within each treatment having different superscripts differ significantly $(P<0.05)$. SEM standard error of the mean

\section{Effect of interactions between biological treatments,} roughage source, and replacement levels $(T \times S \times L)$

There were significantly interaction between biological treatments $(\mathrm{T})$, roughage sources $(\mathrm{S})$, and replacement levels $(\mathrm{L})(\mathrm{T} \times \mathrm{S} \times \mathrm{L})$ only for TBWG and ADG. The best TBWG and ADG were recorded for rabbits fed diets replaced $33 \%$ of $\mathrm{CH}$ by $\mathrm{CS}$ that treated with Trichoderma reesei (1939 g and $21.31 \mathrm{~g}$ ), respectively.

The best fed conversion was realized by rabbits fed diet replaced $\mathrm{CH}$ at 33\% with RS treated by Pleurotus ostreatus (4.05 g DMI/g gain) as shown in Tables 5 and 6.

\section{Economic evaluation}

The profitability of using rice straw or corn stalks as a nonconventional cheap feed stuff in growing rabbit diets depends on the price of these by-products and its effect on growth performance.

Data presented in Table 7 illustrated that the lowest cost price/kg feed (2.136 LE) was recorded with rabbits fed $100 \%$ corn stalks group that is biologically treated with Trichoderma reesei followed by those fed $100 \%$ corn stalks without Trichoderma reesei (2.195 LE) than rabbits group fed diets contained $100 \%$ of RS without Pleurotus ostreatus (2.216 LE).

Results obtained also indicated that group rabbits fed 33\% biologically treated RS with Pleurotus ostreatus showed the highest economic efficiency (179\%) followed by rabbits received 33\% for both RS without Pleurotus ostreatus and rabbits received CS biologically treated with Trichoderma reesei (161\%). Meanwhile, the lowest value of economic efficiency is recorded by rabbits fed diet contained 100\% CS without Trichoderma reesei (75.80\%) followed by rabbits offered diet contained 100\% treated RS without Pleurotus ostreatus (88.00\%).

\section{Discussion}

Data published in Table 1 was in agreement with those found by Shoukry et al. (1985) who noted that fungal

Table 5 Effect of interactions between biological treatments, roughage source and replacement levels $(T \times S \times L)$ on nutrient growth performance of the experimental groups

\begin{tabular}{|c|c|c|c|c|c|c|c|c|c|c|c|c|c|c|}
\hline \multirow[t]{6}{*}{ Item } & \multirow{4}{*}{$\begin{array}{l}\text { Clover } \\
\text { hay } \\
(\mathrm{CH}) \\
\text { Control } \\
0\end{array}$} & \multicolumn{6}{|c|}{ Rice straw (RS) } & \multicolumn{6}{|c|}{ Corn stalks (CS) } & \\
\hline & & \multicolumn{3}{|c|}{ Without } & \multicolumn{3}{|l|}{ With } & \multicolumn{3}{|c|}{ Without } & \multicolumn{3}{|l|}{ With } & \\
\hline & & \multicolumn{3}{|c|}{ Pleurotus ostreatus } & \multicolumn{3}{|c|}{ Pleurotus ostreatus } & \multicolumn{3}{|c|}{ Trichoderma reesei } & \multicolumn{3}{|c|}{ Trichoderma reesei } & \\
\hline & & 33 & 66 & 100 & 33 & 66 & 100 & 33 & 66 & 100 & 33 & 66 & 100 & \\
\hline & $\%$ & $\%$ & $\%$ & $\%$ & $\%$ & $\%$ & $\%$ & $\%$ & $\%$ & $\%$ & $\%$ & $\%$ & $\%$ & \\
\hline & $\mathrm{R}_{1}$ & $\mathrm{R}_{2}$ & $\mathrm{R}_{3}$ & $\mathrm{R}_{4}$ & $\mathrm{R}_{5}$ & $\mathrm{R}_{6}$ & $\mathrm{R}_{7}$ & $\mathrm{R}_{8}$ & $\mathrm{R}_{9}$ & $\mathrm{R}_{10}$ & $\mathrm{R}_{11}$ & $\mathrm{R}_{12}$ & $\mathrm{R}_{13}$ & SEM \\
\hline Initial weight (IW), g & $566^{\mathrm{ef}}$ & $564^{\mathrm{bcd}}$ & $577^{\mathrm{de}}$ & $567^{\mathrm{ef}}$ & $553^{\mathrm{ab}}$ & $570^{b c}$ & $583^{e f}$ & $568^{\text {cde }}$ & $581^{e f}$ & $570^{e f}$ & $576^{a}$ & $554^{\mathrm{abc}}$ & $514^{\mathrm{ef}}$ & 13.57 \\
\hline Final weight (FW),g & 2086 & 2289 & 2151 & 1950 & 2446 & 2335 & 2087 & 2207 & 2055 & 1900 & 2515 & 2344 & 2080 & 21.82 \\
\hline Total body weight gain (TBWG), g & $1528^{9}$ & $1725^{d}$ & $1574^{f}$ & $1383^{i}$ & $1893^{b}$ & $1765^{c}$ & $1504^{\mathrm{g}}$ & $1639^{e}$ & $1474^{h}$ & $1330^{j}$ & $1939^{a}$ & $1790^{c}$ & $1566^{9}$ & 17.55 \\
\hline \multicolumn{8}{|l|}{ Duration period } & & & & \multicolumn{3}{|c|}{91 days } & \\
\hline Average daily gain (ADG), g & $16.70^{9}$ & $18.96^{\mathrm{d}}$ & $17.30^{f}$ & $15.20^{i}$ & $20.80^{\mathrm{b}}$ & $19.40^{c}$ & $16.53^{g h}$ & $18.01^{e}$ & $16.20^{\mathrm{h}}$ & $14.62^{\mathrm{j}}$ & $21.31^{a}$ & $19.67^{c}$ & $17.21^{9}$ & 0.19 \\
\hline Dry matter intake (DMI), g & 88.83 & 82.34 & 89.25 & 89.66 & 83.87 & 87.93 & 85.10 & 92.60 & 93.04 & 89.31 & 94.00 & 89.86 & 95.54 & 1.38 \\
\hline $\begin{array}{l}\text { Feed conversion expressed as (g DM } \\
\text { intake / g gain) }\end{array}$ & $5.32^{\mathrm{ab}}$ & $4.34^{\mathrm{bc}}$ & $5.16^{\mathrm{ab}}$ & $5.90^{\mathrm{a}}$ & $4.05^{\mathrm{c}}$ & $4.59^{\mathrm{bc}}$ & $5.14^{\mathrm{ab}}$ & $5.08^{\mathrm{abc}}$ & $5.80^{\mathrm{a}}$ & $6.08^{\mathrm{a}}$ & $4.43^{\mathrm{bc}}$ & $4.62^{b c}$ & $5.72^{\mathrm{a}}$ & 0.09 \\
\hline
\end{tabular}

$\mathrm{a}, \mathrm{b}, \mathrm{c}, \mathrm{d}, \mathrm{e}, \mathrm{f}, \mathrm{g}, \mathrm{h}, \mathrm{i}$, and $\mathrm{j}$ : means in the same row having different superscripts differ significantly $(P<0.05)$. SEM standard error of the mean 
Table 6 ANOVA results of biological treatments, roughage sources, and replacement levels on growth performance of the experimental groups

\begin{tabular}{|c|c|c|c|c|c|c|c|}
\hline \multirow[t]{2}{*}{ Item } & \multicolumn{3}{|l|}{ Main effects of } & \multicolumn{4}{|c|}{ Interactions } \\
\hline & Treatments $(\mathrm{T})$ & Sources (S) & Levels (L) & $(T \times S)$ & $(T \times L)$ & $(S \times L)$ & $(T \times S \times L)$ \\
\hline Initial weight (IW), g & NS & NS & NS & NS & NS & NS & NS \\
\hline Final weight (FW), g & * & NS & * & NS & * & NS & NS \\
\hline Total body weight gain (TBWG), g & $*$ & * & * & * & * & NS & $*$ \\
\hline Average daily gain (ADG), g & * & * & * & * & * & NS & * \\
\hline Dry matter intake (DMI), g & NS & NS & NS & NS & NS & NS & NS \\
\hline Feed conversion expressed as (g DM intake/g gain) & * & * & * & NS & NS & NS & NS \\
\hline
\end{tabular}

*Significant $(P<05)$. NS not significant

treatment improves the nutritive value of bagasse because mycelia of all fungi had high cellulose enzyme content which converts cellulosic material into single cell protein or microbial protein and decreased OM after incubation with fungi. Also, our results are in agreement with those obtained by Warren (1996) who reported that the microorganisms are efficient degraders of starch and polysaccharides in plant cell walls as carbon and energy reserves in plant because they have the systems for the hydrolysis of polysaccharides to metabolizable products. Moreover, present results are in the range of results obtained by Abd El-Hakim et al. (2006) who showed that biological treatments of RS increased its CP content and decreased CF content compared to untreated one.

Biological treatment of RS caused decrease in NDF, $\mathrm{ADF}, \mathrm{ADL}$, and hemicellulose contents in comparison with either raw or treated RS. Data concerning with cell wall constituents of RS are in harmony with those recorded by Bassuny et al. (2005) and Al Barakel et al. (2007). Also, Fazaeli et al. (2004) observed that fungal treatments (five species of Pleurotus fungi, coded P-21,
P-30, P-41, P-60, and P-90) significantly increased CP and decreased NDF and ADF contents of wheat straw. However, culture P-21 significantly showed lower ability than others to degrade the NDF and ADF contents. Furthermore, Mahrous and Abou Ammou (2005) found that biological treatments of RS with P. funiculosum or S. cerevisiae or both increased CP and ash and decreased DM, OM, CF, NDF, ADF, ADL, cellulose, and hemicellulose contents compared with the untreated rice straw.

OM, CF, NDF, ADF, CP, and ash contents in CS are in the same trend with those recorded by Israilides et al. (1994) who observed that fermentation of sugar beet pulp with semisolid substrate increased CP content by $9.9 \%$ while ash and lignin contents were found to be similar with those of untreated sugar beet pulp. However, Abd EL-Hakim et al. (2006) showed that biological treatments increased CP content and decreased CF content, compared to untreated one. Abou Ammou et al. (2008) and Al-Asfour (2009) found that when sugar beet pulp and wheat straw are treated with Trichoderma reesei, the OM, CF, NDF, and ADF contents were decreased and CP and ash content were increased.

Table 7 Economic efficiency of the experimental groups

\begin{tabular}{|c|c|c|c|c|c|c|c|c|c|c|c|c|c|}
\hline \multirow[t]{4}{*}{ Ingredients } & \multirow{4}{*}{$\begin{array}{l}\text { Clover } \\
\text { hay } \\
\text { (CH) } \\
\text { control } \\
0 \% \\
\mathrm{R}_{1}\end{array}$} & \multicolumn{6}{|c|}{ Rice straw (RS) } & \multicolumn{6}{|c|}{ Corn stalks (CS) } \\
\hline & & \multicolumn{3}{|c|}{ Without Pleurotus ostreatus } & \multicolumn{3}{|c|}{ With Pleurotus ostreatus } & \multicolumn{3}{|c|}{ Without Trichoderma reesei } & \multicolumn{3}{|c|}{ With Trichoderma reesei } \\
\hline & & $33 \%$ & $66 \%$ & $100 \%$ & $33 \%$ & $66 \%$ & $100 \%$ & $33 \%$ & $66 \%$ & $100 \%$ & $33 \%$ & $66 \%$ & $100 \%$ \\
\hline & & $\mathrm{R}_{2}$ & $\mathrm{R}_{3}$ & $\mathrm{R}_{4}$ & $\mathrm{R}_{5}$ & $\mathrm{R}_{6}$ & $\mathrm{R}_{7}$ & $\mathrm{R}_{8}$ & $\mathrm{R}_{9}$ & $R_{10}$ & $\mathrm{R}_{11}$ & $R_{12}$ & $\mathrm{R}_{13}$ \\
\hline Total feed intake /rabbit (kg) & 8.086 & 7.492 & 8.121 & 8.159 & 7.847 & 7.800 & 7.878 & 8.465 & 8.332 & 8.329 & 8.631 & 8.128 & 8.484 \\
\hline Price/kg feed(LE) & 2.360 & 2.325 & 2.302 & 2.216 & 2.326 & 2.322 & 2.246 & 2.329 & 2.323 & 2.195 & 2.339 & 2.284 & 2.136 \\
\hline Feed cost/rabbit (LE) & 19.08 & 17.42 & 18.69 & 18.08 & 18.25 & 18.16 & 17.69 & 19.715 & 19.355 & 18.282 & 20.188 & 18.564 & 18.122 \\
\hline Total cost $(L E)^{A}$ & 30.58 & 28.91 & 30.18 & 29.59 & 29.74 & 29.67 & 29.20 & 31.215 & 30.855 & 29.782 & 31.688 & 30.064 & 29.622 \\
\hline Marketing weight (kg) & 2.086 & 2.289 & 2.151 & 1.949 & 2.446 & 2.336 & 2.087 & 2.207 & 2.055 & 1.900 & 2.515 & 2.344 & 2.080 \\
\hline Cost/kg body weight ( LE) & 14.66 & 12.63 & 14.03 & 15.18 & 12.16 & 12.70 & 13.99 & 14.144 & 15.014 & 15.674 & 12.600 & 12.826 & 12.241 \\
\hline Total revenue $(\mathrm{LE})^{\mathrm{B}}$ & 41.72 & 45.78 & 43.02 & 38.98 & 48.92 & 46.70 & 41.74 & 44.14 & 41.10 & 38.00 & 50.30 & 46.88 & 41.60 \\
\hline Net revenue $(\mathrm{LE})^{1}$ & 11.14 & 16.87 & 12.84 & 9.39 & 19.17 & 17.04 & 12.54 & 12.925 & 10.45 & 8.18 & 18.612 & 16.612 & 11.978 \\
\hline Economic efficiency ${ }^{2}$ & 0.36 & 0.58 & 0.43 & 0.32 & 0.64 & 0.57 & 0.43 & 0.414 & 0.332 & 0.276 & 0.587 & 0.559 & 0.404 \\
\hline Relative economic efficiency & 100 & 161.00 & 117.00 & 88.00 & 179.00 & 159.00 & 119.00 & 113.70 & 90.00 & 75.80 & 161.00 & 153.60 & 111.00 \\
\hline
\end{tabular}

A: including fixed cost (11.50 LE/rabbit). B: assuming that the selling price is (20 LE). 1: net revenue $=(B-A)$. 2: economic efficiency $=(B-A / A)$ 
On the other hand, NFE content of corn stalks as shown in Table 1 was decreased as a result of treatment without or with Trichoderma reesei by 11.95 and $3.82 \%$ compared to raw CS.

Reduction in NFE of treatment without or with Trichoderma reesei could be related to the consumption of these carbohydrates by microorganism as energy source for their growth and multiplications. These results are in agreement with those reported by Martin (1977) who noted that many bacterial genera are capable to utilize steroids as a sole carbon and energy source, thereby degrading steroids completely to carbon dioxide and water.

Variations in chemical analysis of the experimental diets (Tables 2 and 3) may be related to the difference of ingredients used in tested ration formulations and that in harmony with those reported by NRC (1977) and in the range that is obtained by Chahal (1985) who reported that chemical composition of field crop residues contains about 30-40 \% cellulose, 16-27 \% hemicelluloses, and 3-13 \% lignin.

Our results (Table 4) are in agreement with those found by Amber et al. (2004) and Ahmed (2005) who noted that live body weight was improved significantly by addition of Opti-Zyme to ration containing corn stover which replaced $50 \%$ of clover hay $(\mathrm{CH})$ of the control rabbit rations. This improvement may be related to the beneficial effects caused by biological treatment and micro flora (fungi) helping animals to digest the diets containing non-digestible components like roughage and increase the digestion coefficient by this microorganism and influence the growth and function of the gastrointestinal organs. However, Abd El-Hakim et al. (2006) found that rabbits fed diet containing $35 \%$ of $\mathrm{CH}$ recorded the highest FW and TWG $(P<0.05)$ compared to the other groups. In addition, similar results are found by Abd El-Samee (1995) who observed that feed conversion was improved in young rabbits that consumed diet treated with different probiotics in summer period (3.94.1 vs. 4.3). Also, Ahmed (2005) found that rabbits fed both additives (bokashi and Opti-Zyme) showed the best feed conversion value, compared to the control group. Thayalim and Samarasinghe (2002) found that addition of effective microorganism to rabbit diet improved feed conversion. Also our results are in agreement with those of Amber et al. (2004) who noticed that rabbits fed on 5 or $10 \%$ RS treated with $T$. reesei significantly $(P<0.05)$ increased the final weight. Tuikov et al. (1980) found that addition of lactic acid bacteria in calve feeding improved its growth performance. In addition, these results are in agreement with Huang et al. (1990) who reported that body weight of rabbits increased with addition of Trichoderma cellulolytic enzyme for diets containing rice straw. On the other hand, Bonanno et al. (1999) found that adding probiotic to rabbit ration decreased feed intake. El-Badawi et al. (2007) reported that feed intake was not affected when incorporated with treated sugar beet pulp (TSBP) in diet while was significantly $(P<$ $0.05)$ decreased when incorporated with untreated sugar beet pulp (USBP); this results due to high absorbing capacity $(870 \mathrm{ml}$ water/100 g) and swelling capacity (380\% of SBP), causing reducing rate of feed passage (Cheeke, 1987). Ahmed (1998) observed that feeding rabbits on 5 or $10 \%$ RS treated with fungi $T$. reesei significantly $(P<$ 0.05 ) increased daily weight gain. Also, Hicks et al. (1986) observed that adding Lactobacillus acidophilus and Lactobacillus planetarium of cattle ration increased weight gain. Abd-Allah (2007) showed that inclusion of corn cobs treated with T. viride and Saccharomyces cerevisiae at the rate of 20 and $30 \%$ of CFM in lamb rations significantly increased ADG. El-Badawi et al. (2007) reported that ADG was significantly $(P<0.05)$ higher for rabbits fed 25 and 50\% TSBP diets (25.99 and 23.99) than those fed control 25 and 50\% USBP diets (21.50, 21.04, and $17.00 \mathrm{~g} /$ day, respectively). Feed efficiency values were significantly $(P<0.05)$ higher $(4.55$ and 4.63) for rabbits fed 25 and 50\% TSBP than (5.33, 4.86, and $5.30 \mathrm{~kg}$ feed $/ \mathrm{kg}$ gain) rabbits fed control, 25 and $50 \%$ USBP, respectively. Growth performance index (PI) recorded the highest value (70.84) for rabbits fed $25 \%$ TSBP compared with $65.80,52.94,56.99$, and $45.53 \%$ for rabbits fed 50\%TSBP and control 25 and 50\% USBP, respectively. Increase of weight gain and feed efficiency for rabbits fed diets containing TSBP may be due to containing TSBP high content of exogenous enzymes, amino acids, and other secondary metabolites, like vitamins as a result of microorganism activity. Chen et al. (1987) indicated that using $T$. viride as feed additive in rabbit rations increased weight gain. Besides, Ahmed (2003) reported that replacement $50 \%$ of berseem hay by chemically treated CS with urea and biologically treated with T. viride, Cellulomonas cellulans, and Saccharomyces cerevisiae in sheep rations increased ADG compared with control diet. Also, Khalel et al. (2008) showed that there was significant increase in ADG when lambs fed ration contained $10 \%$ jojoba meal treated with $T$. reese $i$ compared with those fed control ration contained untreated jojoba meal. Gippert et al. (1996) showed that ADG was improved when adding probiotic in rabbit ration. Also, El-Badawi et al. (2007) reported that feed efficiency values were significantly $(P<0.05)$ higher $(4.55$ and 4.63) for rabbits fed 25 and 50\% fungal treated sugar beet pulp than $(5.33,4.86$, and $5.30 \mathrm{~kg}$ feed $/ \mathrm{kg}$ gain) rabbits fed control and 25 and 50\% untreated sugar beet pulp, respectively. Feed efficiency for rabbits fed diets containing TSBP may be due to high content of exogenous enzymes, amino acids, and other secondary metabolites, like vitamins as a result of microorganism activity. Generally, the obtained results of economic efficiency 
indicated higher values with RS diets in comparison with corn stalks diets in both treatments.

Our results concerning with economic evaluation are in agreement with those found by Abd El-Hakim et al. (2006) who noted that the highest value of relative economic efficiency was recorded for biologically treated RS with bacteria and fungi ( $30 \%$ replacement $\mathrm{CH})$ compared to control (35\% clover hay). Therefore, partial or complete substitution of $\mathrm{CH}$ in rabbit rations with such agriculture wastes may be useful in Egypt as a tool for least cost ration formulae in rabbit production. Also, results obtained mentioned that the group of rabbits that received diet replaced $33 \%$ of $\mathrm{CH}$ by RS and CS realized the highest economic efficiency values compared with the other diets. These results are in agreement with those obtained by Bassuny et al. (2005) and Abd ElHakim et al. (2006).

\section{Conclusion}

Under conditions as that available throughout carrying this work and from the results obtained, it can be concluded that biological treatments of rice straw by Pleurotus ostreatus or corn stalks by Trichoderma reesei were safe, realizing an improvement in their chemical analysis and improved both daily gain and feed conversion, decreasing the costing of diet formulation and therefore decreasing the price of $1-\mathrm{kg}$ live body weight.

\section{Abbreviations \\ ADF: Acid detergent fiberADGAverage daily gainADLAcid detergent ligninBHBerseem hayCFCrude fiberCHClover hayCPCrude proteinCSCorn stalkDMIDry matter intakeNDFNeutral detergent fiberNFENitrogen free extractOMOrganic matterRHRelative humidityRSRice strawTDNTotal digestible nutrients}

\section{Acknowledgements}

This work was supported by the National Research Centre.

\section{Authors' contributions}

The authors had designed the study, AAAM carried out the field work and lab. analysis, MYE supervised the study, HAAO had carried out the statistical analysis, and RIE had written the manuscript, and SMA had revised the manuscript, AAE had supervised the study, and WSE had finally revised the manuscript and is the corresponding author to the journal. All the authors had read and approved the final manuscript.

\section{Availability of data and materials}

The datasets used and/or analyzed during the current study are available from the corresponding author on reasonable request.

\section{Ethics approval and consent to participate}

Not applicable

\section{Consent for publication}

Not applicable

\section{Competing interests}

The authors declare that they have no competing interests.

\section{Author details}

${ }^{1}$ Animal Production Department, National Research Centre, 33 El-Bohouth Street, P.O: 12622, Dokki, Giza, Egypt. ${ }^{2}$ Animal Production Department, Faculty of Agriculture, Mansoura University, Mansoura, Egypt. ${ }^{3}$ Animal
Reproduction and Artificial Insemination Department, National Research Centre, 33 El-Bohouth Street, P.O: 12622, Dokki, Giza, Egypt.

Received: 23 September 2019 Accepted: 19 November 2019

Published online: 18 December 2019

\section{References}

Abd El-Samee AM (1995) Using some antibiotics and probiotics for alleviating heat stress on growing and doe rabbits in Egypt. World Rabbit Sci 3(2):107111

Abd El-Galil ERI (2008) Effect of bacterial treatments on chemical composition, cell wall constituents and digestibility of rice straw. Egypt J Nutrition and Feeds 11(3):497-510

Abd El-Hakim AS, Shakweer IME, AzOz AA (2006) Effect of using different levels of biologically treated rice straw in growing rabbits diets on growth performance, nutrients digestibility and some physiologies parameters. Egypt J of Rabbit Science 16(2):191-209

Abd-Allah S.A.E. (2007). Biological treatment of some by-products in ruminants feeding. M. Sc. Thesis, Fac. Agric., Al-Azhar University, Egypt.

Abedo A.A. (2011). Prospects of recycling field crop residues in animal feeding strategy. Review Article. pp 7-8. Animal Production Department, National Research Centre. Dokki, Giza, Egypt.

Abo-Eid HA, El-Ashry MA, Khroshed MM, El-Sayes MF (2007) Effect of biological treatment of some crop residues on their nutritive values: 1- effect of biological treatments on recovery rate, chemical composition and in situ disappearance. Egypt J Nutrition and feeds 10(Special Issue):493-508

Abou Ammou FF, Abdel-Khlek TMM, Mahrous AA, El-Shafie MH (2008) Effect of feeding rations containing graded levels of biologically treated wheat straw on carcass characteristics and some blood parameters of growing lambs. Egyptian J Nutrition and feeds 11(3):469-479

Abu Akkada, A.R. (1988). For national strategic for increasing feed stuff in Egypt, 1St National Conference on Role of Scientific Research in Developing Animal Wealth. Academy of Scientific Research and Technology. Cairo, 25-29 Sep.

Ahmed F.G. (1998). Improvement the nutritive value of some roughages used for rabbits feeding. M. Sc. Thesis, Fac. Agric., Ain Shams University, Egypt,

Ahmed F.G. (2005). Optizyme and bokashi in growing rabbit diets contained agricultural roughages. The 4th International Conference on Rabbit Production in Hot Climates Sharm El- Sheikh, Egypt,

Ahmed H.S.S. (2003). Utilization of cotton stalks chemically and biologically treated in sheep rations. M. Sc. Thesis, Fac. Agric., Cairo University Egypt,

Al-Asfour O.N. (2009). Effect of biological treatment on nutritive value of some agricultural by-products. M. Sc. Thesis, Faculty of Agriculture Ain Shams University Egypt.

Al-Barakel F, Jawasreh K, Al-Hamed B (2007) The nutritive value of fungal treated industrial and agricultural by-products and its effect on Awassi sheep performance. Egypt J Nutrition and feeds 10(Special Issue):585-597

Ali A, Azim FA, Bhatti MH (1987) Treatment of cereal straw with rot-fungi. Pak J Agric Res 8(4):428-433

Amber K.H., Yakout H.M. and Hamed R.S (2004). Effect of feeding diets containing yucca extract or probiotic on growth, digestibility, nitrogen balance and caecal microbial activity of growing New Zealand White rabbits In proc. $8^{\text {th }}$ World Rabbit Congress, Puebla, Mexico, 737-741.

AOAC (2005). Official methods of analysis, 18th ed. Association of Official Analytical Chemists, Washington, DC, USA.

Balch C.C. (1976). The potential of poor quality agricultural roughages for animal feeding. F.A.O. Animal Production and Health Paper No.4, New feed resources food and Agriculture Organization of the United Nation.

Bassuny SM, Abdel-Aziz AA, Abd El-Fatah HJ, Abdel-Aziz MYS (2005) Fibrous crop by-product as feed. 4-Effect of biological treatment for rice straw and bean straw on chemical composition, digestibility and some ruminal and blood constituents of sheep. Egypt J Nutrition and Feeds 8(Special Issue):541-554

Bonanno A, Alabiso M, Di Grigoli A, Leto G, Alicata ML (1999) Effect of feeding diets supplemented with Bacillus subtilis and Bacillus licheniformis on the growth performance of rabbits from birth to slaughter. Coniglicoltura 7(8): 47-53

Chahal D.S. (1985). Solid state fermentation with Trichoderma reesei for cellulose production. Appl. Environ. Microbial., 49-205.

Cheeke P.R. (1987). Rabbit feeding and nutrition. Academic press, INC. Orlando, Florida, 32887, U.S.A. pp. 376

Chen XP, Ceng SX, Wang FS, Fu GB, Xing QY, Guo XH, Chen SY, Liu T, Lin CR (1987) Effect of Trichoderma viride leaven, used as a feed additive, on the 
growth of rabbits. Chinese, Journal rabbit, Farming Zhonggoo, Yang Tu, Zazh 3:13-16

Duncan DB (1955) Multiple range and multiple (F-test). Biometrics 11:1-42

El-Badawi A.Y., A.A. Hassan and A.A. Abedo, (2007). Growth performance of NewZealand White rabbits fed diets containing different levels of untreated or fungal treated sugar beet pulp. The 58th EAAP Meeting, August 26-29, 2007. Dublin, Ireland

El-Shinnawy M.M. (1990). The role of fibrous residues in feeding ruminants. $3^{\text {rd }}$ International Symposium on Feed Manufacture and Quality Control, PP. 321-326.

Fadel M, Amra H, Murad HA, Hamed KE (1992) Production of fungal protein from wheat straw by Penicillium funiculosum. Egypt J Food Sci 20:1-13

Fazaeli H, Mahmodzadeh H, Aziz A, Jelan ZA, Liang JB, Rouzbehan Y, Osman A (2004) Nutritive value of wheat straw treated with Pleurotus fungi. AsianAustralian J Anim Sci 17(2):1681-1688

Gippert T, Bersenyi A, Szabo L, Farkas Z (1996) Development of novel feed concentrates supplemented with salinomycin and lacto-sacc for growing rabbit nutrition in small scale farms. 6th World Rabbit Congr. Toulouse (France) 1:187-190

Goering H.K. and Van Soest P.J. (1970). Forage fiber analysis, apparatus, reagents, procedures and some applications. Agriculture handbook No. 379, USDAARS, Washington, DC, P. 20402.

R.B. Hicks, D.R. Gill, R.A. Smith, and R.L. Ball (1986). The effect of a microbial culture on health and performance of newly arrived stocker cattle. Nut Abst and Rev., Vol. 57, Abst.263.

Huang L.G., Y.W. Ning, and J.F. Xi (1990). Studies on microbial fermented rice straw used as rabbit feed. Jcangsu of Journal Agricultural Science, 6 (supplement): 60-65.

Israilides CJ, Icanomou D, Kandylis K, Nikoyris P (1994) Ferment ability of sugar beet and its acceptability in mice. Bioresour Technol 47(2):97-101

Khalel MS, Hassan AA, Shwerab AM, Khayyal AA (2008) Feed evaluation of chemically or biologically treated jojoba meal. Egypt J Nutrition and Feeds 11(3):481-495

Kiran S (1991) Biodegradation of macro-molecules of feeds for livestock. First International Animal Nutrition Workers Conference for Asia and Pacific. September 23-28, Bangalore (India). Compendium 1:92-99

Krishna M, Kamalamma U, Krishnappa (1996) Effect of feeding yeast culture on rumen fermentation in vitro and production performance in cross bred dairy cows. Anim Feed Sci Technol 57:247-256

Mahrous AA, Aabou Ammou FF (2005) Effect of biological treatments of rice straw on sheep productive performance. Egypt J Nutrition and Feeds 8(Special Issue):529-540

Martin D. R. (1977). Effect of buffers upon fiber digestion. On Regulation of AcidBase Balance Symposium. Tucson, Arizona.

Ministry of Agriculture and Lank Reclamation, (2008). Part 1. Published by Agriculture Research Center, Egypt. Ministry of Agriculture.

NRC (1977). National Research Council. Nutrient requirements of rabbits, National Academy of Science, Washington, D.C.

Rangaswami G. and Bagyaraj D.J. (1996). Agricultural microbiology. Second Edition. Prentice-Hall of India, New Delh.

Rangnekar DV, Badve VC, Kharat ST, Sobole BN, Joshi AL (1982) Effect of high pressure steam treatment on chemical composition and in vitro digestibility of roughages. Anim Feed Sci Technol 7:61-70

Rissanen H, Kossila V, Kommeri M, Lampila M (1981) Ammonia treated straw in the feeding of dairy cows and growing cattle. Agric Environ 6:267-271

Sadek Enath E. (2013). Economic and environmental impacts of using agricultural waste for producing non-traditional feeds. Proceeding of the 14th Scientific Conference of Animal Nutrition, 26-29 November, 2013. Hurghada, Egypt. Egyptian J Nutrition and Feeds, Vol.16 (2) Special Issue, pp 139-147.

Shoukry M.M. (2013). An overview on the potentiality of using agricultural byproducts in feeding ruminants. Proceeding of the 14th Scientific Conference of Animal Nutrition, 26-29 Nov 2013, Hurghada, Egypt. Egyptian J. Nutrition and Feeds, Vol.16 (2) Special Issue.

Shoukry MM, Hamissa FA, Ahmed SM, El-Refai AH, Ali HM, Abdel-Motagally ZMZ (1985) Nutritive improvement of some low quality roughages for ruminant. I. Effect of different microbial and chemical treatments on the quality of sugarcance bagasse. Egypt J Anim Prod 25(2):329-342

SPSS (2008). Statistical Package for Social Sciences, Statistics for Windows, Version 17.0. Released 2008. Chicago, U.S.A.: SPSS Inc.

Thayalim S. and Samarasinghe K. (2002). Influence of EM on the performance of the domestic rabbit (oryctolagus cuniculus). 7th International Conference on kyusei Nature Fanning. New Zealand, pp. 7-8.
Tuikov R, Khristova M, Rizvanov K, Vladimitov I (1980) Early weaning of calves using a milk additive and a preparation of freeze-dried bacteria. Zhivotnov dni Nauki 17(7):3-8

Van Soest PJ, Robertson JB, Lewis BA (1991) Methods for dietary fiber, neutral detergent fiber and non starch polysaccharides in relation to animal performance. J Dairy Sci 74:3583-3597

Warren RA (1996) Microbial hydrolysis of polysaccharides. Annu Rev Microbiol 50: $183-212$

\section{Publisher's Note}

Springer Nature remains neutral with regard to jurisdictional claims in published maps and institutional affiliations.

\section{Submit your manuscript to a SpringerOpen ${ }^{\circ}$ journal and benefit from:}

- Convenient online submission

- Rigorous peer review

- Open access: articles freely available online

- High visibility within the field

- Retaining the copyright to your article

Submit your next manuscript at $\boldsymbol{\nabla}$ springeropen.com 